\title{
New parameterization of external and induced fields in geomagnetic field modeling, and a candidate model for IGRF 2005
}

\author{
Nils Olsen ${ }^{1}$, Terence J. Sabaka ${ }^{2}$, and Frank Lowes ${ }^{3}$ \\ ${ }^{1}$ Danish National Space Center, Juliane Maries Vej 30, 2100 Copenhagen, Denmark \\ ${ }^{2}$ Geodynamics Branch, NASA GSFC, Greenbelt/MD, USA \\ ${ }^{3}$ Physics Department, University of Newcastle upon Tyne, United Kingdom
}

(Received December 22, 2004; Revised June 23, 2005; Accepted June 23, 2005)

\begin{abstract}
When deriving spherical harmonic models of the Earth's magnetic field, low-degree external field contributions are traditionally considered by assuming that their expansion coefficient $q_{1}^{0}$ varies linearly with the $D_{\text {st }}$-index, while induced contributions are considered assuming a constant ratio $Q_{1}$ of induced to external coefficients. A value of $Q_{1}=0.27$ was found from Magsat data and has been used by several authors when deriving recent field models from Ørsted and CHAMP data. We describe a new approach that considers external and induced field based on a separation of $D_{\mathrm{st}}=E_{\mathrm{st}}+I_{\mathrm{st}}$ into external $\left(E_{\mathrm{st}}\right)$ and induced $\left(I_{\mathrm{st}}\right)$ parts using a $1 \mathrm{D}$ model of mantle conductivity. The temporal behavior of $q_{1}^{0}$ and of the corresponding induced coefficient are parameterized by $E_{\mathrm{st}}$ and $I_{\mathrm{st}}$, respectively. In addition, we account for baseline-instabilities of $D_{\mathrm{st}}$ by estimating a value of $q_{1}^{0}$ for each of the 67 months of Ørsted and CHAMP data that have been used. We discuss the advantage of this new parameterization of external and induced field for geomagnetic field modeling, and describe the derivation of candidate models for IGRF 2005.
\end{abstract}

Key words: Geomagnetic Reference Model, IGRF/DGRF, magnetospheric currents, induction, spherical harmonic analysis.

\section{Introduction}

It is common practice to consider low-degree external and secondary (induced) field contributions when deriving spherical harmonic models of the Earth's core and crustal fields. Usually, the magnetic field vector $\mathbf{B}=-\nabla V$ is derived from a scalar potential $V$ which is expanded in spherical harmonics:

$$
\begin{aligned}
V= & a \sum_{n, m}\left(g_{n}^{m} \cos m \phi+h_{n}^{m}(t) \sin m \phi\right)\left(\frac{a}{r}\right)^{n+1} P_{n}^{m}(\cos \theta) \\
& +a \sum_{n, m}\left(q_{n}^{m} \cos m T_{m}+s_{n}^{m} \sin m T_{m}\right)\left(\frac{r}{a}\right)^{n} P_{n}^{m}\left(\cos \theta_{d}\right) \\
& +a D_{s t}(t) \cdot \hat{q}_{1}^{0}\left[\left(\frac{r}{a}\right)+Q_{1}\left(\frac{a}{r}\right)^{2}\right] P_{1}^{0}\left(\cos \theta_{d}\right) \cdot
\end{aligned}
$$

$(r, \theta, \phi)$ are Earth-centered spherical coordinates (radius, colatitude, longitude), with a reference Earth radius of $a=$ $6371.2 \mathrm{~km}$. $\left(\theta_{d}, T_{m}\right)$ are dipole-colatitude and magnetic local time (MLT). $P_{n}^{m}$ are the Schmidt semi-normalized associated Legendre functions of degree $n$ and order $m$.

The first term of the equation describes internal (core and crustal field) contributions; $\left\{g_{n}^{m}(t), h_{n}^{m}(t)\right\}$ are the corresponding internal Gauss coefficients. They are static (typically for higher degree $n$, describing the crustal field), or slowly varying with time $t$ (for lower degree $n$ ) to account for the secular variation of the core field. The

Copyright (c) The Society of Geomagnetism and Earth, Planetary and Space Sciences (SGEPSS); The Seismological Society of Japan; The Volcanological Society of Japan; The Geodetic Society of Japan; The Japanese Society for Planetary Sciences; TERRAPUB. second term describes external (magnetospheric) contributions; $\left\{q_{n}^{m}, s_{n}^{m}\right\}$ are the corresponding external field coefficients. Since the magnetospheric ring-current (probably the most important magnetospheric contribution) flows in a plane perpendicular to the dipole axis (rather than the geographic axis), we use dipole/MLT-coordinates $\left(\theta_{d}, T_{m}\right)$ to describe external contributions. (In our particular application, the coefficients of the second line of Eq. (1) are assumed constant, and their coefficients for $m=0$ could be presented in the same coordinate system as the first line.) The last term of the above equation takes care of the time changes of the large-scale magnetospheric field and its internal, induced, counterpart. Their time dependence is assumed to be that of the $D_{\text {st }}$-index, with $\hat{q}_{1}^{0}$ as factor of proportionality, and $Q_{1}$ as a constant ratio of induced to external fields. This parameterization was introduced by Langel et al. (1980) and Langel and Estes (1985a, b) and is now common practice in geomagnetic field modeling. For simplicity, only the coefficient with $n=1, m=0$ is considered here (i.e., it is assumed that the field is axially symmetric); terms with order $m=1$ are in practice often considered, too. Their inclusion is straightforward.

Parameterizing both external (inducing) and internal (induced) fields with $D_{\text {st }}(t)$ using a constant value of $Q_{1}$, as done here, is problematic, since the same time dependence is used for both field constituents (and hence any time lag between induced and inducing fields is neglected). We will discuss the approximations made to obtain this term, and present an approach that relaxes the assumptions.

In addition to these approximations, baseline-instability 

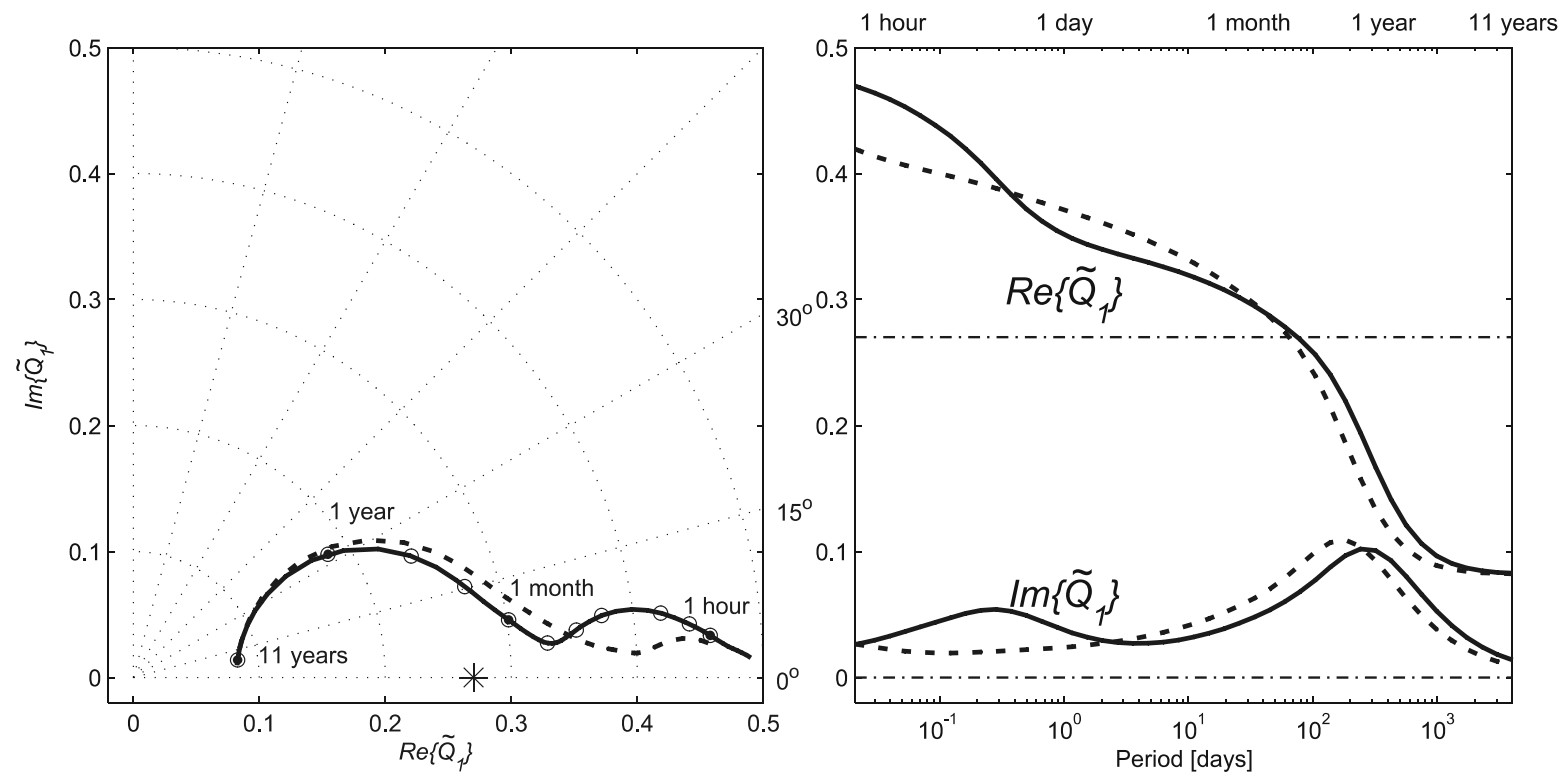

Fig. 1. $Q$-response of various models of mantle conductivity. The solid and dashed curves present values from realistic conductivity models representative of continental and oceanic regions, respectively. Left: polar plot of the real vs. imaginary part of $\widetilde{Q}_{1}$. Right: Dependency of real and imaginary part of $\widetilde{Q}_{1}(\omega)$ on period $T=2 \pi / \omega$. The frequency-independent value $Q_{1}=0.27$ is indicated by a star (left), resp. a dashed-dotted line (right).

in $D_{\text {st }}$ is one of the obstructions to improved field models, and the existence of correlated low-latitude residuals in Ørsted and CHAMP residuals when using the above described approach has been clearly demonstrated (Holme et al., 2003). The coefficient $q_{1}^{0}$ in Eq. (1) is a measure of the strength of the axisymmetric part of the magnetospheric field for $D_{\text {st }}=0 \mathrm{nT}$, so that baseline-instabilities in $D_{\text {st }}$ will result in a time-varying coefficient $q_{1}^{0}$. In the present paper we allow for long-period baseline-instabilities in $D_{\text {st }}$ by estimating a value of $q_{1}^{0}$ for each month.

There are three main current systems contributing to the magnetospheric field (Kivelson and Russell, 1995): magnetopause currents flowing on the magnetospheric boundary (magnetopause), tail currents in the neutral sheet of the geomagnetic tail, and the ring current flowing in the equatorial plane around the Earth. Of these, the ring-current is closest to Earth (distance 3-5a). Its geometry is therefore determined more by that of Earth's main field compared to magnetopause and -tail currents (located at distances $>8 a$ ) which are more influenced by the geometry of the solar wind and the Sun-Earth connection line. Hence it is advantageous to use different coordinate systems for describing the various magnetospheric contributions.

Dipole/MLT coordinates are identical to solar magnetic (SM) coordinates; they are suitable for describing the magnetic effect of the ring-current (cf. Eq. (1)), in agreement with the approach taken for the Tsyganenko models of the magnetosphere (e.g. Tsyganenko, 1990, 2002a, b). However, contributions from magnetopause and tail currents are better described in the solar magnetospheric (GSM) coordinate system (Kivelson and Russell, 1995), which is tilted by the tilt angle $\psi$ with respect to the dipole axis (i.e., the SM $z$-axis). Using GSM coordinates for describing (a part of) magnetospheric contributions in geomagnetic field modeling was introduced by Maus et al. (2005a).
Note, however, that the magnetic field observations contain contributions from several external current systems; it is therefore not possible to discriminate the various magnetospheric contributions by analysis of the magnetic field at a specific time. In the following we start by modelling magnetospheric contributions in general using only the SM system; the additional use of the GSM system will be described in Section 4.

\section{Large-Scale Magnetospheric Variations and Their Induced Counterpart}

Four assumptions have to be made in deriving the last part of Eq. (1). The first assumption is that the spatial structure of the magnetospheric field can be described by spherical harmonics of degree $n=1$ and order $m=0$ (again, inclusion of terms with $m>0$ is straightforward but will not be considered here). Hence the scalar potential describing the external field is given by

$$
V^{\mathrm{e}}(t)=a \epsilon_{1}^{0}(t)\left(\frac{r}{a}\right) P_{1}^{0}\left(\cos \theta_{d}\right)
$$

where $\epsilon_{1}^{0}$ is the expansion coefficient of the external field and the superscript "e" stands for "external".

The second assumption is that the time change of the magnetospheric field is proportional to that of the $D_{\mathrm{st}^{-}}$ index: $\epsilon_{1}^{0}(t)=\hat{q}_{1}^{0} D_{\text {st }}(t)$.

Time change of this primary (magnetospheric) field induces secondary currents in the conducting Earth's interior. In the general case of a conductivity that varies in radial and horizontal direction (3-D conductivity), these induced currents produce magnetic field variations that may contain all spherical harmonic degrees and orders. In other words: although the primary, magnetospheric, field is of $P_{1}^{0}$ geometry, the secondary, induced, field contains contributions $\iota_{n}^{m}$ for all $n, m$. However, if we make our third assumption, 
that conductivity depends only on depth (1-D conductivity), each external coefficient induces only one internal coefficient of same degree $n$ and order $m$. In that case the scalar potential describing external and induced fields (indicated by the superscript "e+i") is

$$
V^{\mathrm{e}+\mathrm{i}}(t)=a\left\{\epsilon_{1}^{0}(t)\left(\frac{r}{a}\right)+\iota_{1}^{0}(t)\left(\frac{a}{r}\right)^{2}\right\} P_{1}^{0}\left(\cos \theta_{d}\right)
$$

In the frequency domain (time dependency $e^{i \omega t}$, where $\omega$ is angular frequency), this equation becomes

$$
\widetilde{V}^{\mathrm{e}+\mathrm{i}}(\omega)=a\left\{\widetilde{\epsilon}_{1}^{0}(\omega)\left(\frac{r}{a}\right)+\widetilde{\iota}_{1}^{0}(\omega)\left(\frac{a}{r}\right)^{2}\right\} P_{1}^{0}\left(\cos \theta_{d}\right)
$$

where the tilde " $\sim$ " denotes a quantity in the frequency domain.

The assumption of a 1-D conductivity leads to a coupling of the induced $\left(\iota_{1}^{0}\right)$ and inducing $\left(\epsilon_{1}^{0}\right)$ coefficients. In the frequency domain the dependency is linear:

$$
\widetilde{\iota}_{1}^{0}(\omega)=\widetilde{Q}_{1}(\omega) \widetilde{\epsilon}_{1}^{0}(\omega)
$$

where $\tilde{Q}_{1}(\omega)$ is the so called $Q$-response (see Schmucker, $1985 \mathrm{a}, \mathrm{b}, 1987$ for a discussion of its properties). Multiplication in the frequency domain corresponds to a convolution in the time domain:

$$
\iota_{1}^{0}(t)=Q_{1} \star \epsilon_{1}^{0}=\int_{-\infty}^{\infty} Q_{1}\left(t-t^{\prime}\right) \epsilon_{1}^{0}\left(t^{\prime}\right) d t^{\prime}
$$

where the asterisk “ $\star$ " indicates convolution. Combining Eqs. (4) and (5) yields

$$
\widetilde{V}^{\mathrm{e}+\mathrm{i}}(\omega)=a \widetilde{\epsilon}_{1}^{0}(\omega) \cdot\left\{\left(\frac{r}{a}\right)+\widetilde{Q}_{1}(\omega)\left(\frac{a}{r}\right)^{2}\right\} P_{1}^{0}\left(\cos \theta_{d}\right)
$$

which corresponds in the time domain to

$$
\begin{aligned}
V^{\mathrm{e}+\mathrm{i}}(t)= & a\left\{\epsilon_{1}^{0}(t)\left(\frac{r}{a}\right)+\left(\frac{a}{r}\right)^{2} \int_{-\infty}^{\infty} Q_{1}\left(t-t^{\prime}\right) \epsilon_{1}^{0}\left(t^{\prime}\right) d t^{\prime}\right\} \\
& \cdot P_{1}^{0}\left(\cos \theta_{d}\right)
\end{aligned}
$$

The last assumption made when deriving the third part of Eq. (1) is that mantle conductivity belongs to a very special case of 1-D models, consisting of an insulating upper mantle and a superconductor below depth $d$ (i.e., below radius $r=c=a-d)$. In that case the $Q$-response is independent of frequency $\omega$ and is given by

$$
\widetilde{Q}_{1}=\frac{1}{2}\left(\frac{c}{a}\right)^{3}
$$

which leads to $\widetilde{Q}_{1}=0.27$ for a superconductor below $d=1200 \mathrm{~km}$ depth. Since a frequency-independent value of $\widetilde{Q}_{1}$ corresponds to a delta function in the time domain, the convolution in Eq. (8) results in a multiplication:

$$
\begin{aligned}
V^{\mathrm{e}+\mathrm{i}}(t) & =a \epsilon_{1}^{0}(t) \cdot\left\{\left(\frac{r}{a}\right)+Q_{1}\left(\frac{a}{r}\right)^{2}\right\} P_{1}^{0}\left(\cos \theta_{d}\right) \\
& =a \hat{q}_{1}^{0} \cdot D_{\mathrm{st}}(t) \cdot\left\{\left(\frac{r}{a}\right)+Q_{1}\left(\frac{a}{r}\right)^{2}\right\} P_{1}^{0}\left(\cos \theta_{d}\right)(10)
\end{aligned}
$$

where $\widetilde{Q}_{1}$ has been replaced by $Q_{1}$ for simplicity. This equation is identical to the last part of Eq. (1). The value $Q_{1}=0.27$ was found by Langel and Estes (1985a) from an analysis of Magsat data and has been widely used for deriving field models from Ørsted and CHAMP (e.g., Olsen, 2002; Olsen et al. 2000; Holme et al., 2003).

However, $Q$-responses calculated from realistic models of mantle conductivity are rather different. The solid lines of Fig. 1 shows the values obtained from the mantle conductivity model of Schmucker (1985b), which is representative for continental areas. The dashed lines are the response of "model B" of Utada et al. (2003); this model is representative for oceanic regions. The frequency independent value of $Q_{1}=0.27$ is shown by a dash-dotted line. The comparatively small difference between the solid and the dashed curves may be regarded as an indication of the error introduced by assuming 1-D mantle conductivity, but it is obvious that a constant value of $Q_{1}=0.27$ (star, resp. dash-dotted line) is a rather crude approximation.

The zero imaginary part (and hence zero phase) of $Q_{1}=$ 0.27 indicates that there is no time-lag between external and induced fields for the superconductor/insulator conductivity model. In contrast, the greater than zero phase of the realistic $Q$-responses indicates that the induced field lags the external field. However, since the imaginary part of $\tilde{Q}_{1}(\omega)$ is generally much smaller than its real part, the time lag is small.

More important than the non-zero phase is the change of amplitude, $\left|\tilde{Q}_{1}\right|$, with frequency. For the realistic responses, $\left|\tilde{Q}_{1}\right|$ is larger than 0.27 for excitation periods shorter than a few months or so, but much smaller for longer periods. The induced part of long-period magnetospheric signals is therefore much more attenuated, compared to shorter periods; the mantle acts as a high-pass filter. (This is contrary to the attenuation of the core field secular variation while penetrating through the real conducting mantle, for which the mantle acts as a low-pass filter.) As a consequence, external and induced fields have a different temporal behavior and should not be parameterized by the same index $D_{\mathrm{st}}(t)$. An alternative approach is described below.

\section{Decomposition of the $D_{\mathrm{st}}$-Index into External and Induced Part}

$D_{\text {st }}$ is the north-component of the axially symmetric part of the equatorial disturbance field at the Earth's surface caused by the magnetospheric ring-current and its induced counterpart. Hence $D_{\text {st }}$ contains both external and induced contributions,

$$
D_{\mathrm{st}}(t)=E_{\mathrm{st}}(t)+I_{\mathrm{st}}(t)
$$

The magnetic north-component at the equator at $r=a$ is found from Eq. (3) to be $D_{\text {st }}=X=-\partial V /(a \partial \theta)=$ $-\left(\epsilon_{1}^{0}+\iota_{1}^{0}\right)$, and comparison with Eq. (11) yields $E_{\mathrm{st}}=-\epsilon_{1}^{0}$ and $I_{\mathrm{st}}=-\iota_{1}^{0}$.

$E_{\text {st }}$ and $I_{\text {st }}$ can be separated with the aid of the $Q$ response calculated from a given mantle conductivity model. Such a decomposition has been proposed independently by Maus and Weidelt (2004) and Olsen (2004) and may be done in the following way: $D_{\mathrm{st}}(t)$ is Fourier transformed to obtain $\widetilde{D}_{\text {st }}(\omega)$, and the response $\tilde{Q}_{1}(\omega)$ is calcu- 

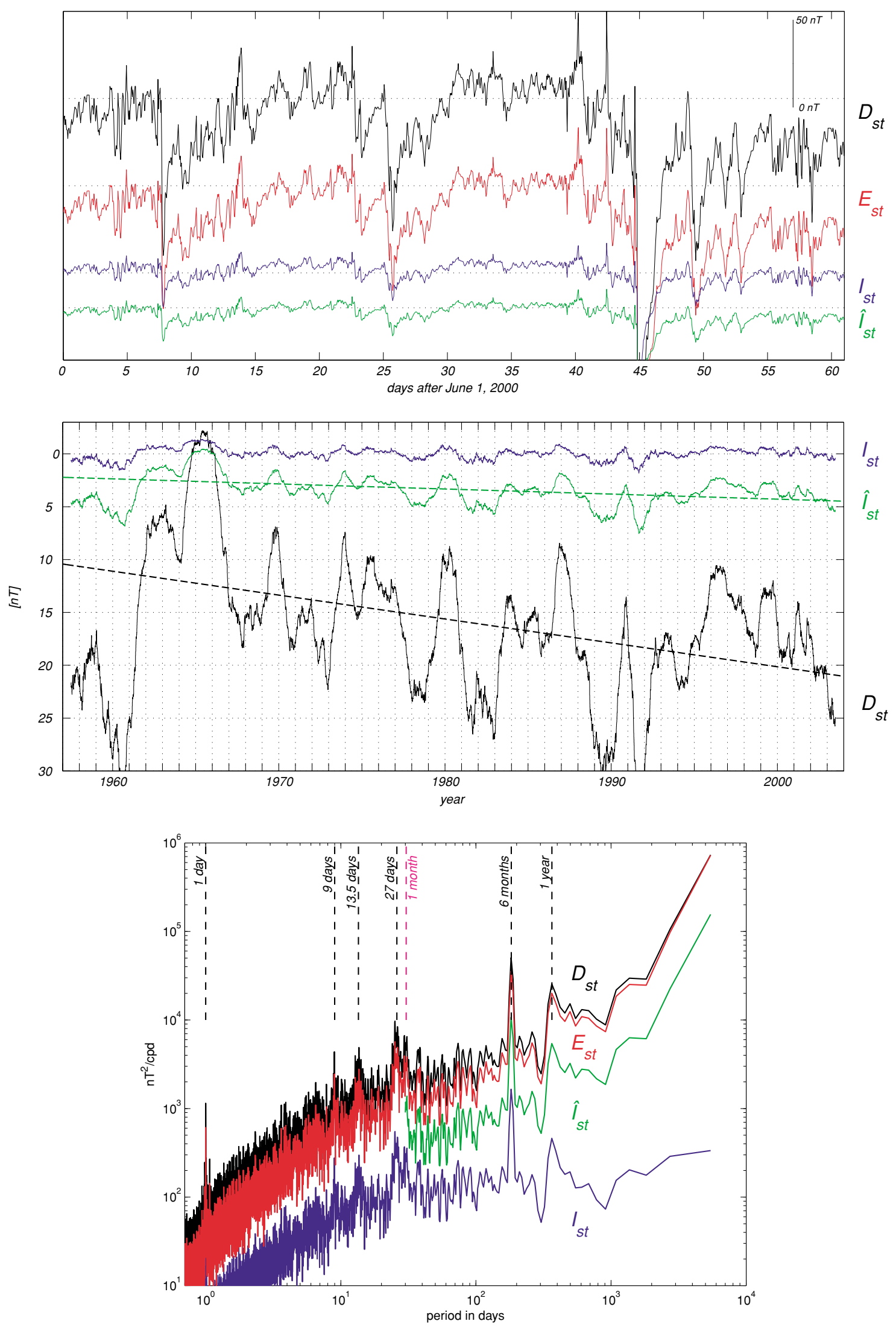

Fig. 2. Top: Time series of $D_{\text {st }}$ (black) $E_{\text {st }}$ (red) and $I_{\text {st }}$ (blue), for June and July 2000 . The green curve shows $\hat{I}_{\mathrm{st}}=D_{\text {st }} \cdot Q_{1}\left(/ 1+Q_{1}\right)=0.21 D_{\text {st }}(t)$; the value traditionally used for describing the time-changes of induced contributions. Bottom: One-year moving averages for the years $1957-2004$. The dashed lines present linear trend estimates. Bottom: Power spectrum (in units of nT ${ }^{2} / \mathrm{cpd}$, where cpd stands for "cycles per day") of $D_{\mathrm{st}}, E_{\mathrm{st}}, \hat{I}_{\mathrm{st}}$ and $I_{\mathrm{st}}$, obtained from hourly mean values of the years 1957-2003. The spectrum for $\hat{I}_{\mathrm{st}}$ is only shown for periods larger than 1 month. 
lated for all considered frequencies using a given model of mantle conductivity. Next

$$
\widetilde{I}_{\mathrm{st}}(\omega)=\frac{\widetilde{Q}_{1}(\omega)}{1+\widetilde{Q}_{1}(\omega)} \widetilde{D}_{\mathrm{st}}(\omega)
$$

is determined using Eqs. (5) and (11). Finally, $\widetilde{I}_{\mathrm{st}}(t)$ is transformed back to the time domain to obtain $I_{\mathrm{st}}(t)$, and $E_{\mathrm{st}}(t)=D_{\mathrm{st}}(t)-I_{\mathrm{st}}(t)$ is calculated.

As an example, the top panel of Fig. 2 shows two months of $D_{\text {st }}$ (black curve) and its constituents $E_{\text {st }}$ (red) and $I_{\text {st }}$ (blue), separated using the conductivity model of Utada et al. (2003). (It is expected that use of a different, but realistic, conductivity model only results in small changes.) The dotted lines indicate the respective zero-levels. Separation of $D_{\text {st }}$ using a constant (frequency independent) value of $Q_{1}$ yields $D_{\mathrm{st}}(t)=\hat{E}_{\mathrm{st}}(t)+\hat{I}_{\mathrm{st}}(t)$ with

$$
\begin{aligned}
& \hat{E}_{\mathrm{st}}(t)=\frac{1}{1+Q_{1}} D_{\mathrm{st}}(t) \\
& \hat{I}_{\mathrm{st}}(t)=\frac{Q_{1}}{1+Q_{1}} D_{\mathrm{st}}(t),
\end{aligned}
$$

which results, for $Q_{1}=0.27$, in $\hat{E}_{\mathrm{st}}(t)=0.79 D_{\mathrm{st}}(t)$, $\hat{I}_{\mathrm{st}}(t)=0.21 D_{\mathrm{st}}(t) . \quad \hat{I}_{\mathrm{st}}$, shown by the green curve, is a proxy for $I_{\mathrm{st}}(t)$, for the case of a constant $Q_{1}$.

Proper handling of long-term variations in the induced fields (whether real or spurious) is essential for deriving good field models; errors will influence estimates of the main-field coefficient $g_{1}^{0}$ and its time change. The presence of long-term fluctuations in $D_{\text {st }}$ can be studied by looking at 1-year moving averages, presented in the middle part of Fig. 2. The black curve shows the 1-year average of $D_{\text {st }}$; the blue and green curve present in the same way processed $I_{\mathrm{st}}$ and $\hat{I}_{\mathrm{st}}$. Applying a one-year moving average smooths much of the time-changes of $D_{\text {st }}$; however, the smoothed time-series reveals a large number of spikes, steps and excursions, which occur preferentially at the turn of the years. This is due to the way the index is calculated (definition of the baseline by polynomials over a few years) and indicates that the baseline of $D_{\text {st }}$ might not be constant in time.

Note the non-zero offset of $D_{\text {st }}$. This offset, though reduced in size, is also present in $\hat{I}_{\mathrm{st}}$, due to the assumed proportionality with $D_{\mathrm{st}}$. However, a static induced field (offset) is unphysical, and hence its presence indicates an error that will be introduced by assuming a constant value of $Q_{1}$. Using proper (frequency dependent) values of the $Q$-response, the offset disappears, as indicated by the zero mean of $I_{\text {st }}$.

The bottom panel of Fig. 2 shows the power spectra of the various indices. The well known periodicities of geomagnetic activity (annual and semi-annual periods, 27-day solar rotation period and its harmonics, daily period) are clearly seen. Surprisingly, there is also a peak at a period of 1 month (indicated by the magenta vertical line), which may be an artifact introduced during the calculation of $D_{\text {st }}$. The induced part, $I_{\mathrm{st}}$, obtained using a realistic Earth model has more than 10 times (100 times) less power for periods longer than 1 year ( 8 years) compared to the signal obtained with a fixed $Q$-value (i.e., $\hat{I}_{\text {st }}$ ), because $I_{\text {st }}$ is much less influenced by baseline instabilities in $D_{\text {st }}$ than is $\hat{I}_{\text {st }}$.

Since $\hat{I}_{\mathrm{st}}$ is traditionally used to parameterize internal (induced) contributions, the data are forced to fit an unphysical internal dipole field of a few nT size. This results in main field coefficients $g_{1}^{0}$ that are biased (too low) by a value of equal size but opposite sign.

Moreover, there is an obvious trend of about $-0.15 \mathrm{nT} / \mathrm{yr}$ in $D_{\text {st }}$, and a somewhat smaller trend in $\hat{I}_{\text {st }}$ (see middle panel of Fig. 2). However, the trend is much larger during the last years, and when data covering only the last five years are analyzed (Ørsted and CHAMP period), the trend is about $-1 \mathrm{nT} / \mathrm{yr}$ in $D_{\mathrm{st}}$ and about $-0.2 \mathrm{nT} / \mathrm{yr}$ in $\hat{I}_{\mathrm{st}}$. Use of $\hat{I}_{\mathrm{st}}$ to parameterize internal fields will therefore result in a secular variation (SV) coefficient $\dot{g}_{1}^{0}$ that is biased (too low) by about $0.2 \mathrm{nT} / \mathrm{yr}$; this is confirmed by field modeling, as demonstrated in the next section.

Separation of $D_{\text {st }}$ into external and induced parts allows us to parameterize external and induced field contributions in a more appropriate way compared to the usual approach shown in the last term of Eq. (1) and Eq. (10). We instead parameterize external fields by $E_{\mathrm{st}}(t)$ and induced fields by $I_{\mathrm{st}}(t)$, and use

$V^{\mathrm{e}+\mathrm{i}}(t)=a \check{q}_{1}^{0}(t) \cdot\left\{E_{\mathrm{st}}(t)\left(\frac{r}{a}\right)+I_{\mathrm{st}}(t)\left(\frac{a}{r}\right)^{2}\right\} P_{1}^{0}\left(\cos \theta_{d}\right)$

instead of the last line of Eq. (1), where both the external and induced fields are taken proportional to $D_{\text {st }}(t)$.

\section{Application: Estimation of New Field Models}

The above-described new parameterization of external and induced fields has been applied to more than 5 years of satellite data from the Ørsted and CHAMP satellites. Data selection and model parameterization follows closely that of previous models like the OSVM (Olsen, 2002).

We use Ørsted scalar and vector data between March 1999 and September 2004, and CHAMP scalar data between August 2000 and August 2004. We use the $K p$ index to restrict the data to quiet times, specifically requiring $K p \leq 1+$ for the time of observation and $K p \leq 20$ for the previous three hour interval. Contrary to previous models, we do not select data according to the absolute value of the $D_{\text {st }}$ index (since the baseline of $D_{\text {st }}$ is not constant; for instance during the first half of $2003 D_{\text {st }}$ is probably off by $15 \mathrm{nT}$, as discussed later). However, we require that $D_{\text {st }}$ does not change by more than $1 \mathrm{nT} / \mathrm{hr}$. Only data from dark regions (sun $5^{\circ}$ below horizon) were used, to reduce contributions from ionospheric currents at middle and low latitudes. The effect of polar cap ionospheric currents is minimized by excluding data in the polar caps for which the dawn-dusk component of the interplanetary magnetic field was $\left|B_{y}\right|>3 \mathrm{nT}$. Ørsted vector data have been taken for dipole latitudes equatorward of $\pm 60^{\circ}$, scalar data were used for regions poleward of $\pm 60^{\circ}$ or if attitude data were not available. Sampling interval was 60 seconds; weights $w \propto \sin \theta$ are applied, to simulate an equal-area distribution. Ørsted vector data show anisotropic errors due to attitude uncertainty from calculating orientation from only one star camera, this is explicitly modeled in the inver- 
Table 1. Number $N$ of data points as well as mean and rms (in nT) for the four derived models:

Oersted(09a/04): derived using the "classical" approach of describing induced contributions (Eq. (10))

Oersted(09b/04): as Oersted(09a/04), but derived using the new approach of describing induced contributions (Eq. (14))

Oersted(09c/04): as Oersted(09b/04), but solving for a time-varying $q_{1}^{0}(t)$

Oersted(09d/04): as Oersted(09c/04), but including the two external coefficients $q_{1}^{0, G S M}$ and $q_{2}^{0, G S M}$ in GSM coordinates

\begin{tabular}{|c|c|c|c|c|c|c|c|c|c|c|}
\hline \multirow{3}{*}{ Parameters } & \multirow[b]{3}{*}{ Component } & \multirow[b]{3}{*}{$N$} & \multirow{2}{*}{\multicolumn{2}{|c|}{$\frac{\operatorname{Oersted}(09 \mathrm{a} / 04)}{1467}$}} & \multirow{2}{*}{\multicolumn{2}{|c|}{$\frac{\text { Oersted }(09 \mathrm{~b} / 04)}{1467}$}} & \multirow{2}{*}{\multicolumn{2}{|c|}{$\frac{\text { Oersted }(09 \mathrm{c} / 04)}{1533}$}} & \multirow{2}{*}{\multicolumn{2}{|c|}{$\begin{array}{c}\text { Oersted }(09 d / 04) \\
1535\end{array}$}} \\
\hline & & & & & & & & & & \\
\hline & & & mean & $\mathrm{rms}$ & mean & $\mathrm{rms}$ & mean & $\mathrm{rms}$ & mean & rms \\
\hline \multirow[t]{2}{*}{ all } & $F_{\text {polar }}$ & 54,880 & 0.01 & 5.31 & -0.03 & 4.69 & -0.01 & 4.54 & 0.01 & 4.53 \\
\hline & $F_{\text {nonpolar }}+B_{B}$ & 156,214 & 0.05 & 3.95 & 0.06 & 3.18 & 0.06 & 2.95 & 0.05 & 2.71 \\
\hline \multirow[t]{4}{*}{ Ørsted } & $F_{\text {polar }}$ & 32,272 & 0.50 & 4.86 & 0.51 & 4.24 & 0.56 & 4.02 & 0.57 & 4.02 \\
\hline & $F_{\text {nonpolar }}+B_{B}$ & 96,915 & 0.41 & 3.29 & 0.43 & 3.04 & 0.41 & 2.82 & 0.41 & 2.57 \\
\hline & $B_{\perp}$ & 61,878 & -0.24 & 7.90 & -0.18 & 7.92 & -0.22 & 7.73 & -0.26 & 7.61 \\
\hline & $B_{3}$ & 64,725 & -0.18 & 4.09 & -0.01 & 4.07 & -0.10 & 3.94 & -0.17 & 3.70 \\
\hline \multirow[t]{5}{*}{ CHAMP } & $F_{\text {polar }}$ & 22,608 & -0.79 & 5.97 & -0.89 & 5.32 & -0.91 & 5.27 & -0.88 & 5.25 \\
\hline & $F_{\text {nonpolar }}+B_{B}$ & 59,299 & -0.52 & 3.68 & -0.54 & 3.40 & -0.52 & 3.15 & -0.55 & 2.92 \\
\hline & $g_{1}^{0}[\mathrm{nT}]$ & & \multicolumn{2}{|c|}{$-29,591.6$} & \multicolumn{2}{|c|}{$-29,587.9$} & \multicolumn{2}{|c|}{$-29,587.8$} & \multicolumn{2}{|c|}{$-29,587.8$} \\
\hline & $\dot{g}_{1}^{0}[\mathrm{nT} / \mathrm{yr}]$ & & \multicolumn{2}{|c|}{12.1} & \multicolumn{2}{|r|}{12.3} & \multicolumn{2}{|r|}{12.3} & \multicolumn{2}{|r|}{12.3} \\
\hline & $\ddot{g}_{1}^{0}\left[\mathrm{nT} / \mathrm{yr}^{2}\right]$ & & \multicolumn{2}{|c|}{-0.70} & \multicolumn{2}{|c|}{-0.94} & \multicolumn{2}{|c|}{-0.91} & & -0.90 \\
\hline
\end{tabular}

sion (Holme and Bloxham, 1996; Holme, 2000). Since we are mostly interested in spherical harmonic coefficients describing core and long-wavelength lithospheric fields, we have subtracted the short-wavelength $(n>30)$ lithospheric field as given by CM4 (Sabaka et al., 2004) from all observations.

Following Eq. (1), the time dependence of the internal Gauss coefficients $\left\{g_{n}^{m}(t), h_{n}^{m}(t)\right\}$ are expanded in a Taylor series according to

$$
\begin{aligned}
& g_{n}^{m}(t)=g_{n}^{m}+\left(t-t_{0}\right) \cdot \dot{g}_{n}^{m}+\frac{1}{2}\left(t-t_{0}\right)^{2} \cdot \ddot{g}_{n}^{m} \text { for } n=1-8 \\
& =g_{n}^{m}+\left(t-t_{0}\right) \cdot \dot{g}_{n}^{m} \\
& =g_{n}^{m} \quad \text { (const.) }
\end{aligned}
$$

and similar for the coefficients $h_{n}^{m}(t) . t_{0}=2002.0$ is model epoch. This yields 1088 static coefficients (up to degree and order $N_{\text {static }}=32$ ), 288 coefficients of secular variation (up to $N_{\mathrm{SV}}=16$ ), and 80 coefficients of secular acceleration (up to $N_{\mathrm{SA}}=8$ ).

As for the OSVM, external coefficients $\left\{q_{n}^{m}, s_{n}^{m}\right\}$ are estimated up to degree $N_{\text {ext }}=2$ (which gives 8 coefficients). However, contrary to previous models we use dipole-colatitude/MLT coordinates, $\theta_{d}, T_{m}$ (instead of colatitude and longitude).

Several models were derived, using the same data and (almost the same) number of model parameters. The only difference between the first two models is that the classical approach of treating magnetospheric and induced fields using $D_{\text {st }}$ and a fixed value $Q_{1}=0.27$ (Eq. (10), but for $m=0,1)$ was used for deriving the first model, called Oersted(09a/04) while for the second model, called Oersted(09b/04), the new approach (Eq. (14), but for $m=0,1$ ) was used, i.e. external fields are parameterized by $E_{\text {st }}$ while their internal, induced counterparts are parameterized by $I_{\mathrm{st}}$. In both cases, 3 coefficients of proportionality $\left(\hat{q}_{1}^{0}, \hat{q}_{1}^{1}, \hat{s}_{1}^{0}\right.$, or $\left.\check{q}_{1}^{0}, \check{q}_{1}^{1}, \check{s}_{1}^{0}\right)$ are estimated.

In total, each model has 1467 free parameters (1456 internal coefficients describing the static field, secular variation and secular acceleration, 8 static external coefficients, 3 coefficients of $D_{\mathrm{st}} / E_{\mathrm{st}} / I_{\mathrm{st}}$-dependency).

Table 1 shows the statistics of the models. The new parameterization (Eq. (14)) reduces the model misfit by up to $25 \%$ (for the scalar misfit at non-polar latitudes), compared to using the "classical" approach (Eq. (10)). Note that the number of parameters is the same for both models. Also listed are the values of the main coefficient $g_{1}^{0}$ and of its first and second time derivative. $g_{1}^{0}$ of the second model, Oersted(09b/04), is 3.7 nT less negative than the corresponding value of model Oersted(09a/04), and $\dot{g}_{1}^{0}$ is larger by $0.2 \mathrm{nT} / \mathrm{yr}$, in agreement with the predictions made in the previous section.

The top part of Fig. 3 presents the low-latitude scalar residuals as a function of time, for model Oersted(09a/04). (The residuals of model Oersted(09b/04) are similar.) The Ørsted and CHAMP residuals are shown in grey and black, respectively, where zero levels are indicated by the horizontal lines. The time parameterization of the model is clearly insufficient, as there is considerable time-dependent signal remaining in the residuals, in agreement with the findings of Holme et al. (2003). Further, the residuals are strongly correlated between Ørsted and CHAMP, and so it is unlikely that they result from differences in local time of the satellites orbits. The most probable explanation is the existence of base-line instabilities in $D_{\mathrm{st}}$. There is for instance a depletion between day count 1100 and 1300 (i.e. the first half of 2003) of 10 to $20 \mathrm{nT}$, indicating that the $D_{\text {st }}$ baseline is probably too low by a comparable amount. Note that the final version of the $D_{\text {st }}$ index was used before January 2003; the provisional index was used between January 2003 and February 2004, and the "near realtime" version of $D_{\text {st }}$ was 


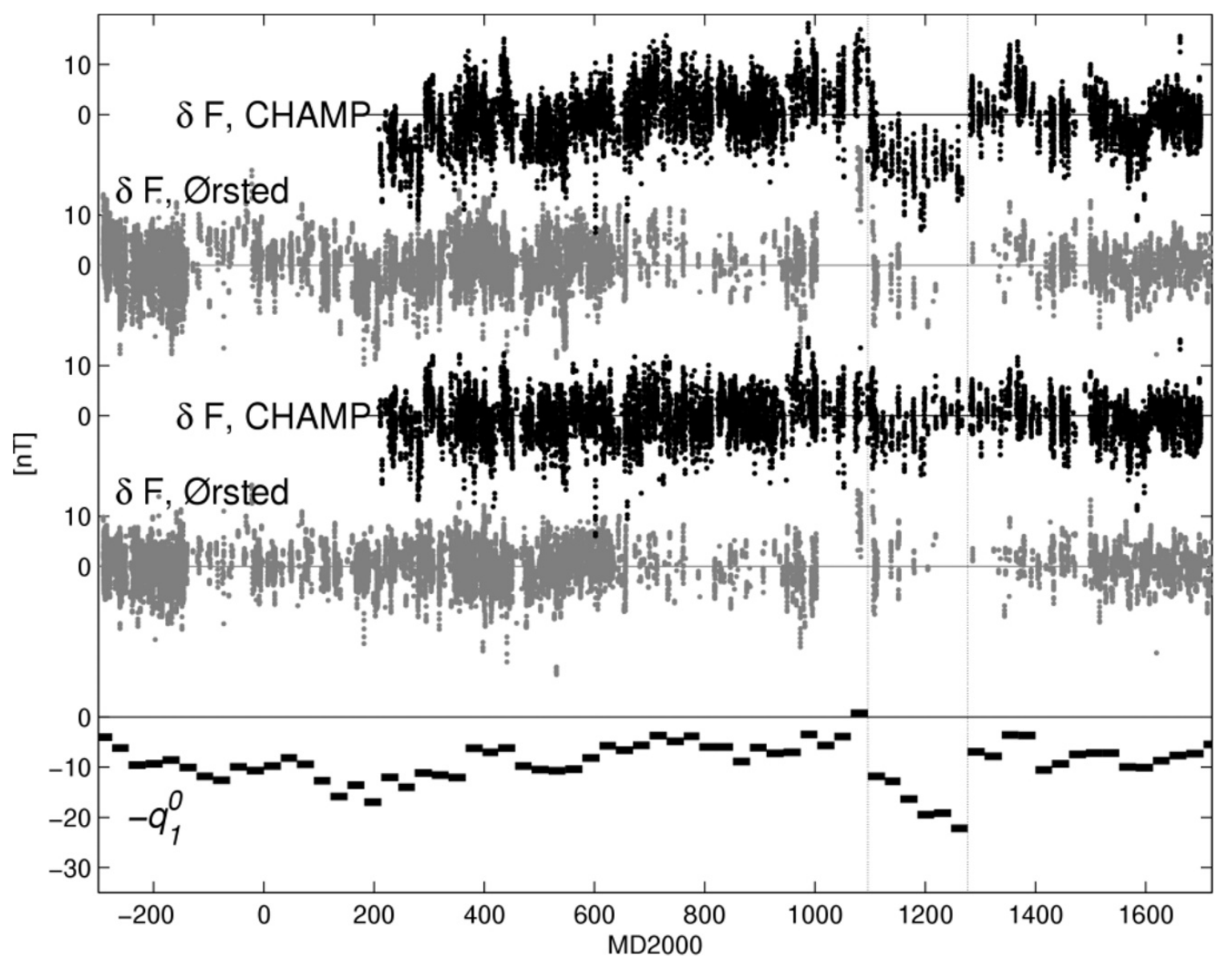

Fig. 3. Low-latitude $\left(< \pm 10^{\circ}\right.$ dip-latitude) scalar residuals as a function of time (day after midnight, 1/1/2000) with respect to model Oersted(09a/04) (upper part) and Oersted(09d/04) (middle part). Also shown is $-q_{1}^{0}(t)$ of model Oersted(09d/04) (lower part). Horizontal lines represent zero-levels.

used after March 1, 2004. The observed large offset after day count 1100 is probably because the final $D_{\text {st }}$ index was not available for 2003 onward.

To investigate this, we derived a third model, called Oersted(09c/04) where we allow the "static" external coefficient $q_{1}^{0}$ (cf. the second term of Eq. (1)) to change with time. We do this by estimating 67 values of $q_{1}^{0}$, one for each month of the data window (March 1999-September 2004). The lower part of Fig. 3 shows these values of $-q_{1}^{0}$, together with time series of the low-latitude scalar residuals with respect to that model. (At the equator, the magnetic intensity of a $P_{1}^{0}$ source field is proportional to $-q_{1}^{0}$, which is the reason for choosing the negative sign.) There is a considerable variation; the time-change of $q_{1}^{0}$ suggests for instance that $D_{\text {st }}$ is off by about $15 \mathrm{nT}$ during the first 6 months of 2003 (indicated by the dashed vertical lines). The misfit of that model is slightly smaller (reduction of non-polar scalar misfit of 7\%) compared to the second model; however, one has to keep in mind that the number of model parameters has increased by $66(4 \%)$. Co-estimation of a $D_{\text {st }}$ offset on a monthly basis, as done here, removes most of the correlated low-latitude residuals, as can be seen when comparing the two upper and the middle part of Fig. 3.

Contrary to previous models like OSVM, the present models do not incorporate annual and semi-annual variations (solving for an annual and semi-annual variation of $q_{1}^{0}$ would be in conflict with the explicit determination of monthly values for $q_{1}^{0}$, described in the previous paragraph). However, as mentioned before, currents in the magnetopause and tail are better described in the GSM coordinate system; a field which is constant in GSM coordinates has seasonal and daily variations in an Earth-fixed coordinate system. To investigate the effect of including GSM-coefficients we derived a fourth model, called Oersted(09d/04). It is identical to model Oersted(09c/04) but includes also the two coefficients $q_{1}^{0, \mathrm{GSM}}$ and $q_{2}^{0, \mathrm{GSM}} \mathrm{de}$ scribing daily and seasonal field variations, as seen in the dipole coordinate system, of a magnetospheric field which is constant in the GSM system. The total number of parameters of that model is thus 1535 (1456 static internal coefficients; 7 static external coefficients in the SM coordinate system; 67 external SM coefficients $q_{1}^{0}$ (one for each month); 3 coefficients of $I_{\text {st }} / E_{\mathrm{st}}$ dependency; 2 external GSM-coefficients). Table 1 shows that the model misfit of that last model is further decreased (scalar misfit at nonpolar latitudes is about $9 \%$ lower compared to model Oersted(09c/04)), although that last model only solves for two additional parameters.

\section{Discussion}

Lowes-Mauersberger power spectra of, and degree correlation (equation 4.23, Langel and Hinze, 1998) between, various models are presented in Fig. 4. Its left part shows the spectra of the static field of models Oersted(09a/04) and 

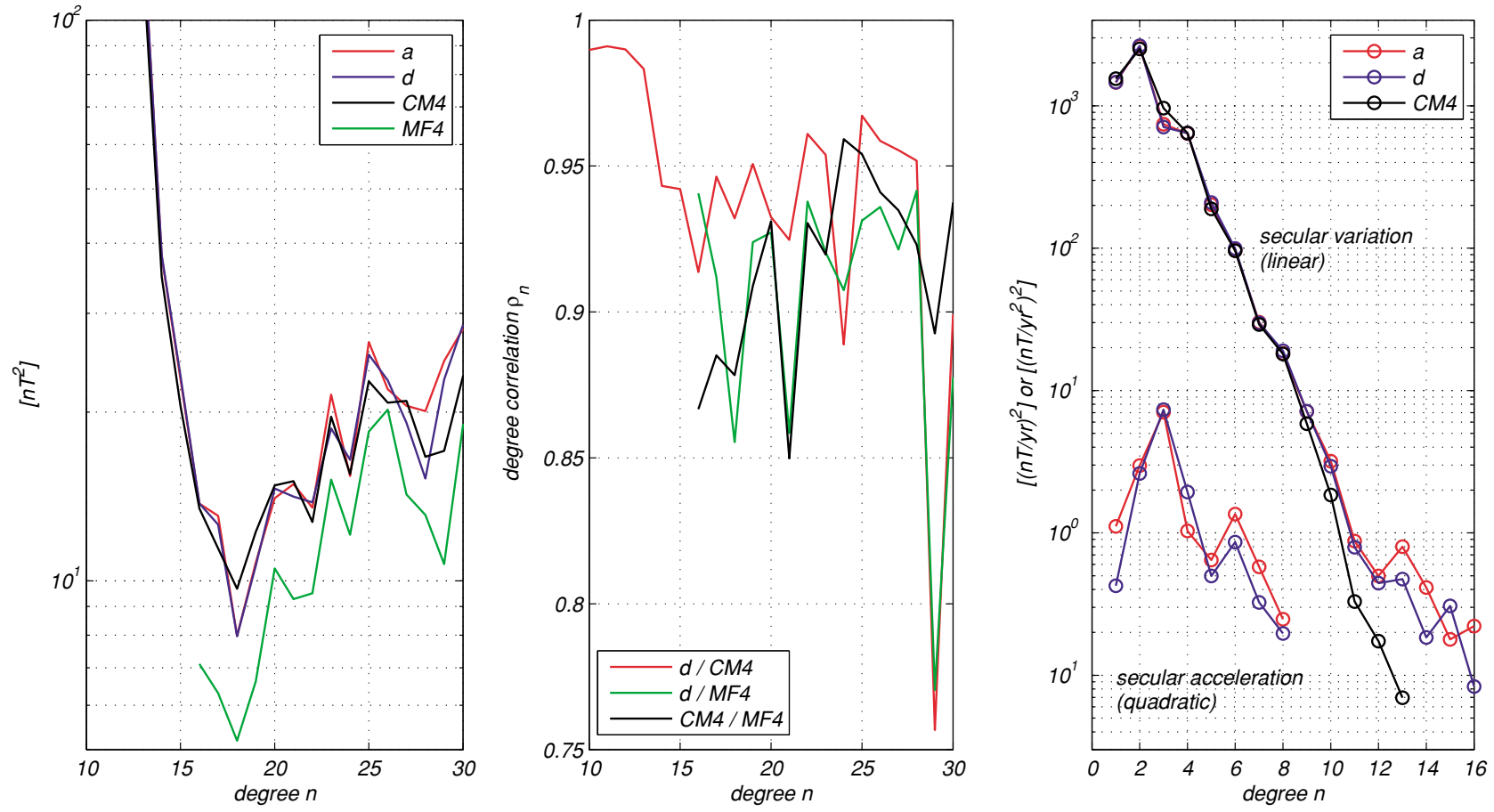

Fig. 4. Left: Power spectra of the static field part of various models. Middle: Degree correlation between the static part of the models. Right: Power spectra of secular variation and secular acceleration, respectively.

09d in comparison with that of CM4 (Sabaka et al., 2004) and MF4 (Maus et al., 2005b). Note that MF4 is a model of the crustal field only, and therfore contains only coefficients with degree $n \geq 16$. The three models 09a, 09d and CM4 have roughly the same power, while MF4 has considerably less power, partly due to the use of a track-by-track filtering of the data when deriving MF4.

The middle panel shows degree correlation, $\rho_{n}$, between the static part of the models 09d, CM4 and MF4. There is consistently higher correlation between models $09 \mathrm{~d}$ and CM4 compared to MF4. The right panel shows the power spectra of the linear and quadratic time changes (secular variation and acceleration, respectively). With the exception of secular acceleration at degree $n=1$, model $09 \mathrm{~d}$ contains less power compared to model 09a, which may indicate that the coefficients of model 09a are more strongly influenced by unmodeled signals than those of model 09d. Also shown is secular variation of CM4 for epoch 2002.0. Note that CM4 is regularized, which is the reason for the faster decrease of power above degree 8 or so.

The complexity of modeling magnetospheric contributions increases from model Oersted(09a/04) to model 09d, and it is interesting to investigate how this affects $q_{1}^{0}$, the main coefficient of magnetospheric contributions. The static coefficient $q_{1}^{0}$ changes from $20.2 \mathrm{nT}$ to $16.7 \mathrm{nT}$ between model 09a and 09b; this change is probably mainly due to use of $E_{\text {st }}$ instead of $D_{\text {st }}$ (which should decrease the coefficient by a factor of 0.79 ). The mean value of the (now time-dependent) coefficient $q_{1}^{0}$ of model $09 \mathrm{c}$ is $\overline{q_{1}^{0}}=17.2 \mathrm{nT}$, in good agreement with the value of model 09b. As for model 09d, the sum of the magnetospheric contributions in SM and GSM coordinates is very similar to that value: $\overline{q_{1}^{0}}+g_{1}^{0, \mathrm{GSM}}=17.4 \mathrm{nT}$; however, about one half is constant wrt the dipole (SM) coordinate system $\left(\overline{q_{1}^{0}}=9.1 \mathrm{nT}\right)$, while the other half is constant in the GSM coordinate system $\left(g_{1}^{0, \mathrm{GSM}}=8.3 \mathrm{nT}\right)$. All other external coefficients have amplitudes well below $1 \mathrm{nT}$, with the exception of $q_{2}^{1}$, which is between $-1.1 \mathrm{nT}$ (model 09b) and $-1.5 \mathrm{nT}$ (model 09d).

As pointed out by Maus et al. (2005a) and Maus and Lühr (2005), modeling magnetospheric fields in the GSM coordinate system introduces some seasonal and daily variations in an Earth-fixed system, due to the time changes of the dipole-tilt $\psi$. Secondary currents are therefore induced in the Earth's interior which, however, are not considered in the presented models; they are expected to have amplitudes below $1 \mathrm{nT}$

\section{Extraction of the IGRF 2005 Candidate Models}

Our candidate model IGRF-A 1 is an $N=13$ truncation of model Oersted(09d/04), propagated to epoch 2005.0 (using linear as well as quadratic terms). The same correction for ionospheric leakage that we used for our candidate model for DGRF2000 (cf., Olsen et al., 2005), based on the work of Lowes and Olsen (2004), has been applied, and the formal standard deviations of the coefficients have been scaled by the same empirical factors.

Two candidate models for secular variation have been derived from model Oersted(09d/04): SV-A1-2005 is the $N=8$ truncation of the secular variation after propagation to epoch 2005.0, while SV-A3-2007.5 is the $N=8$ truncation of the secular variation after propagation to epoch 2007.5. Both propagations were performed using linear as well as quadratic terms.

The spectrum of secular acceleration shown in the right 
panel of Fig. 4 peaks at degree $n=3$; this peak is due to a rather large value for the coefficient $\ddot{h}_{3}^{3}=1.2 \mathrm{nT} / \mathrm{yr}^{2}$. For the extraction of the IGRF candidates we have to extrapolate the coefficients from model epoch $t_{0}=2002.0$ to epoch 2005.0 (for the main field) and 2007.5 (for the secular variation), respectively. Since a quadratic extrapolation is problematic, we decided to derive an additional model, Oersted(09g/04), without quadratic terms. Only data from one year (August 2003-September 2004) were used; data selection and model parameterization is similar to that used for model Oersted(09a/04), but the model epoch is 2005.0, truncation level of the secular variation is $N_{S V}=13$, and no quadratic time terms were derived $\left(N_{S A}=0\right)$. The $N=13$ truncation of that model, after correcting for ionospheric leakage, is our second main field candidate, called IGRFA2. Likewise, another secular variation candidate, SV-A22005 , is derived as the $N=8$ truncation of the secular variation of model Oersted( $09 \mathrm{~g} / 04)$. However, we derived these two candidate models (IGRF-A2 and SV-A2-2005) mainly for evaluation purposes; candidate model IGRF-A1 is our preferred candidate for the main field, and SV-A3-2007.5 is our preferred candidate for secular variation.

\section{References}

Holme, R., Modelling of attitude error in vector magnetic data: application to Ørsted data, Earth Planets Space, 52, 1187-1197, 2000.

Holme, R. and J. Bloxham, The treatment of attitude errors in satellite geomagnetic data, Phys. Earth Planet. Int., 98, 221-233, 1996.

Holme, R., N. Olsen, M. Rother, and H. Lühr, CO2: A CHAMP magnetic field model, in First CHAMP Mission results for Gravity, Magnetic and Atmospheric Studies, edited by C. Reigber, H. Lühr, and P. Schwintzer, pp. 220-225, Springer Verlag, 2003.

Kivelson, M. G. and C. T. Russell, Introduction to Space Physics, Cambridge University Press, 1995.

Langel, R. A. and R. H. Estes, Large-scale, near-Earth magnetic fields from external sources and the corresponding induced internal field, $J$. Geophys. Res., 90, 2487-2494, 1985a.

Langel, R. A. and R. H. Estes, The near-Earth magnetic field at 1980 determined from MAGSAT data, J. Geophys. Res., 90, 2495-2509, 1985 b.

Langel, R. A. and W. J. Hinze, The Magnetic Field of the Earth's Lithosphere: The Satellite Perspective, Cambridge University Press, 1998.

Langel, R. A., G. D. Mead, E. R. Lancaster, R. H. Estes, and E. B. Fabiano, Initial geomagnetic field model from Magsat vector data, Geophys. Res. Lett., 7, 793-796, 1980.

Lowes, F. J. and N. Olsen, A more realistic estimate of the variances and systematic errors in spherical harmonic geomagnetic field models, Geophys. J. Int., 157, 1027-1044, 2004.
Maus, S. and H. Lühr, Signature of the quiet-time magnetospheric magnetic field and its electromagnetic induction in the rotating Earth, Geophys. J. Int., 162, 755-763, 2005.

Maus, S. and P. Weidelt, Separating the magnetospheric disturbance magnetic field into external and transient internal contributions using a 1D conductivity model of the Earth, Geophys. Res. Lett., 31, L12,614, doi:10.1029/2004GL020,232, 2004.

Maus, S., H. Lühr, G. Balasis, M. Rother, and M. Mandea, Introducing POMME, the POtsdam Magnetic Model of the Earth, in Earth Observation with CHAMP, Results from Three Years in Orbit, edited by C. Reigber, H. Lühr, P. Schwintzer, and J. Wickert, pp. 293-298, Springer Verlag, 2005a.

Maus, S., M. Rother, K. Hemant, H. Lühr, A. V. Kuvshinov, and N. Olsen, Earth's crustal magnetic field determined to spherical harmonic degree 90 from CHAMP satellite measurements, Geophys. J. Int., 2005b (submitted).

Olsen, N., A model of the geomagnetic field and its secular variation for epoch 2000 estimated from Ørsted data, Geophys. J. Int., 149, 454-462, 2002.

Olsen, N., New parameterization of external and induced fields in geomagnetic field modeling, Geophysical Research Abstracts, 6, 02,454, 2004.

Olsen, N., T. J. Sabaka, and L. Tøffner-Clausen, Determination of the IGRF 2000 model, Earth Planets Space, 52, 1175-1182, 2000.

Olsen, N., F. Lowes, and T. J. Sabaka, Ionospheric and induced field leakage in geomagnetic field models, and derivation of candidate models for DGRF 1995 and DGRF 2000, Earth Planets Space, 57, this issue, 1191-1196, 2005.

Sabaka, T. J., N. Olsen, and M. Purucker, Extending comprehensive models of the Earth's magnetic field with Ørsted and CHAMP data, Geophys. J. Int., 159, 521-547, doi: 10.1111/j.1365-246X.2004.02,421.x, 2004.

Schmucker, U., Magnetic and electric fields due to electromagnetic induction by external sources, in Landolt-Börnstein, New-Series, 5/2b, pp. 100-125, Springer-Verlag, Berlin-Heidelberg, 1985a.

Schmucker, U., Electrical properties of the Earth's interior, in LandoltBörnstein, New-Series, 5/2b, pp. 370-397, Springer-Verlag, BerlinHeidelberg, $1985 \mathrm{~b}$.

Schmucker, U., Substitute conductors for electromagnetic response estimates, PAGEOPH, 125, 341-367, 1987.

Tsyganenko, N. A., Quantitative models of the magnetospheric magnetic field: methods and results, Space Sci.Rev., 54, 75-186, 1990.

Tsyganenko, N. A., A model of the near magnetosphere with a dawndusk asymmetry 1. Mathematical structure, J. Geophys. Res., 107, 12-1, 2002a.

Tsyganenko, N. A., A model of the near magnetosphere with a dawn-dusk asymmetry 2. Parameterization and fitting to observations, J. Geophys. Res., 107, 10-1, 2002b.

Utada, H., T. Koyama, H. Shimizu, and A. D. Chave, A semi-global reference model for electrical conductivity in the mid-mantle beneath the north Pacific region, Geophys. Res. Lett., 30, 43-1, DOI 10.1029/2002GL016,092, 2003.

N. Olsen (e-mail: nio@spacecenter.dk), T. J. Sabaka, and F. Lowes 